\title{
Bilateral Proptosis in a Case of Recurring Multiple Myeloma: Uncommon Orbital Presentation of Plasmacytoma
}

This article was published in the following Dove Press journal: International Medical Case Reports Journal

\section{Daniel Barmas-Alamdari (iD Guneet S Sodhi Teresa A Shenouda}

Department of Ophthalmology, Eastern Virginia Medical School, Norfolk, VA, USA
Correspondence: Daniel Barmas-

Alamdari

Tel +l 703-537-9932

Email barmasd@evms.edu

\begin{abstract}
Multiple myeloma is a malignant plasma cell dyscrasia that may invade the orbits in extramedullary presentations. Common manifestations of orbital involvement include unilateral proptosis, injection, chemosis, diplopia, and occasionally pain. Additionally, the soft-tissue tumors associated with multiple myeloma typically cause bony destruction of adjacent structures. However, in certain patients, bilateral proptosis and a lack of bony destruction may occur. In these instances, it is important for physicians to consider patient history, presentation, and progression in order to ensure that a potentially fatal diagnosis is not missed. The reason this case is important is two-fold: 1) we describe a case of an individual with relapsing multiple myeloma presenting as bilateral proptosis and lacking bony destruction, highlighting the importance of understanding the disease process and 2) we discuss why patients with multiple myeloma may be more prone to developing tumors of the orbit in recurrent cases.
\end{abstract}

Keywords: multiple myeloma, bilateral proptosis, bony destruction, relapse, sanctuary site

\section{Introduction}

Multiple myeloma is a malignant proliferation of plasma cells that is most active in the bone marrow, but may present with extramedullary findings. The disease is typically defined as having elevated levels of $\mathrm{M}$ protein, an antibody that may cause bone tumors, kidney dysfunction, and above all impaired immune function. Orbital involvement in multiple myeloma is uncommon, having been reported in the literature as less frequently involved compared to other extramedullary sites. ${ }^{1,2}$ A literature review in 2009 suggests less than $50 \%$ of cases present with orbital manifestations as the initial presentation, and approximately $10 \%$ of cases have orbital findings as the primary indication of recurrence. ${ }^{2}$ The most common orbital findings include proptosis (classically unilateral), decreased visual acuity, diplopia, chemosis, ptosis, injection, restricted extraocular movements (EOM) and, more infrequently, pain. ${ }^{2-4}$ Bilateral proptosis in multiple myeloma is also reported, though less prevalent, ${ }^{2,5,6}$ A systemic review conducted by Payandeh et al, focusing on orbital multiple myeloma characteristics from 2009 to 2018, found only 15\% (3/20) of the documented cases of orbital multiple myeloma presented with bilateral findings. ${ }^{7}$ Additionally, Thuro et al documented a series where $17 \%$ of 30 cases of orbital plasmacytomas were bilateral, and $24 \%$ were discrete, non-bone involving. ${ }^{8}$ We describe a case to add to the previously noted literature of recurrent multiple myeloma presenting with bilateral 
proptosis secondary to multiple well-defined soft-tissue tumors not associated with adjacent bony destruction. The collection and evaluation of protected patient health information were HIPAA compliant.

\section{Case Report}

A 45-year-old-male presented to the ophthalmology consult service for bilateral, severe orbital proptosis associated with pain and photophobia. His past medical history was significant for prior diagnosis of multiple myeloma. The patient had been initially diagnosed with multiple myeloma several years prior and was treated with six cycles of thalidomide, dexamethasone, and bortezemib. He relapsed two years after his initial diagnosis, having a testicular mass confirmed to be plasmacytoma following orchiopexy, and a left clavicular mass plasmacytoma, which received radiation therapy. A supraglottic mass was also present at this time, but was determined to be an inflammatory lesion with biopsy showing HSV-1 and was appropriately treated with valacyclovir. He received a regimen of six cycles of cytoxan, dexamethasone, and bortezemib, and then was given a VDT-Pace regimen (bortezomib, dexamethasone, thalidomide, cisplatin, doxorubicin, cyclophosphamide, and etoposide) three times over the course of the next six months.

At his initial presentation to the hematology oncology service, four years after initial multiple myeloma diagnosis, he had significant fever and malaise. The hematology/ oncology service diagnosed him with a multiple myeloma recurrence given his past medical history and lab findings of elevated monoclonal lambda light chains and elevated IgG. He was subsequently admitted for an aggressive chemotherapy regimen. He was noted to have worsening eye redness and was started on naphazoline/pheniramine drops by the primary medicine team. After one week, the ophthalmology service was consulted due to worsening proptosis, lid ecchymosis, and conjunctival hyperemia.

Ophthalmic examination revealed a visual acuity of $20 / 80$ bilaterally with severely limited extraocular movements $(\mathrm{EOM})$ in the right eye (OD), and mildly limited lateral movement in the left eye (OS). Intraocular pressures (IOP) were $22 \mathrm{mmHg}$ bilaterally. There was marked bilateral proptosis and chemosis was greater on the right than left. Severe mechanical ectropion also resulted, though not apparent in the image, and her significant chemosis resulted in exposure keratoconjunctivitis OD with inferior corneal breakdown (Figure 1).

A CT brain and orbits revealed multiple large orbital masses suggestive of metastases. There was also a hyperattenuating extra-axial mass in the right temporal lobe surrounded by vasogenic edema. In this immunocompromised patient, superimposed orbital cellulitis was also considered as part of the differential diagnosis. Broad spectrum antibiotics (IV Vancomycin and Zosyn) were initiated. He was also started on IV Acyclovir to cover for HSV, given the findings of a suspicious temporal lobe lesion and a remote history of having a suspicious esophageal mass that was biopsied and confirmed to be HSV. MRI of the orbits, one

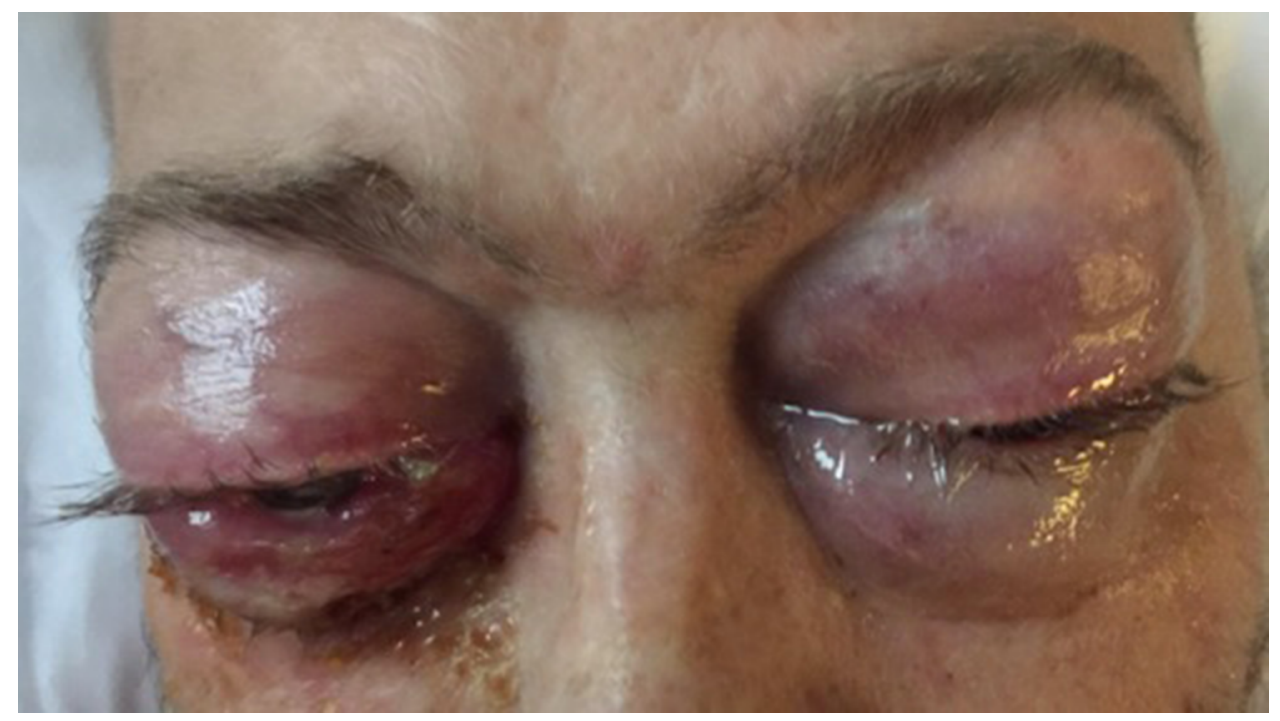

Figure I Photograph showing bilateral ocular proptosis with lid erythema, edema, and severe chemosis, worse in the right eye. This led to lagophthalmos and corneal exposure. 
week post-CT, revealed bilateral enhancing intraorbital masses, resulting in bilateral proptosis with mass effect on the right globe and optic nerve with no compromise in the orbital bone architecture (Figure 2A-C). At this stage, his repeat ophthalmic exam revealed worsening symptoms. His vision was 20/400 bilaterally, a 2-3+ afferent pupillary defect developed in the right eye, and his IOP was now 28 OD and 30 OS. Given these findings, there was a concern for orbital compartment syndrome. Due to the patient's delirious and mentally waxing and waning state, a bedside bilateral canthotomy and cantholysis was not felt to be safe. The definitive surgical plan was to enlarge the orbit via removal of one to four walls with incision into the periosteum, allowing for softtissue prolapse and possible anterior tumor debulking. However, the medicine team did not feel the patient would survive general anesthesia given his immunocompromised and septic state, which included fever, hypotension, and metabolic encephalopathy.

An urgent right anterior orbitotomy and lateral canthotomy/cantholysis under monitored anesthesia care were performed in an effort to prevent progressive optic neuropathy and obtain tissue for biopsy. Pathologic exam demonstrated diffuse plasma cell infiltrates, strongly positive for CD38, CD138, and CD56, with monoclonal lambda expression, highly supportive of multiple myeloma. His visual acuity continued to decline and the patient's mental status became severely altered. Though the oncology service recommended external beam radiotherapy as the treatment of choice, the patient's clinical status continued to decline, at which point he was transitioned to hospice and comfort care. The patient expired shortly afterwards.

\section{Discussion}

Orbital plasma cell dyscrasias typically present with unilateral solitary soft-tissue intraorbital tumors that are extensions of bony deposits and associated with bone destruction. ${ }^{2-6,9,10}$ In this case, the patient presented with bilateral proptosis and multiple, well-defined soft-tissue tumors that were not associated with adjacent bony destruction, which has been a less prevalent presentation in the literature. ${ }^{5,8,11,12}$

Orbital lymphoproliferative disorders (OLPDs) account for around $25 \%$ of all orbital tumors. ${ }^{13}$ The most common type of orbital tumor is lymphoma, while plasmacytomas account for $3 \%$ of orbital tumors. ${ }^{13,14}$ Of these plasmacytomas, Thuro et al reported in an extensive review, that up to $97 \%$ may be associated with multiple myeloma. ${ }^{8}$ This is an important consideration, as plasma cell tumors display similar clinical presentations as OLPDs, but their treatments may vary. Additionally, some lesions may be composed of cells that combine properties of both lymphocytes and plasma cells.

Ophthalmic manifestations of multiple myeloma may present early or late in the disease process and are extensive in nature. Presentations can include direct infiltrates, compression or displacement of tissues and nerves, hyperviscosity syndrome leading to retinopathy, diplopia, visual disturbances, and light chain deposition in ocular tissues. ${ }^{15}$ Symptoms may present similarly in various types of extramedullary plasmacytomas, and can include an insidious onset and slow infiltration, complicating approaches to treatment. ${ }^{2}$ Survival rates of individuals presenting with extramedullary plasmacytomas are low, regardless of the tissue in which the tumors present. However, those with

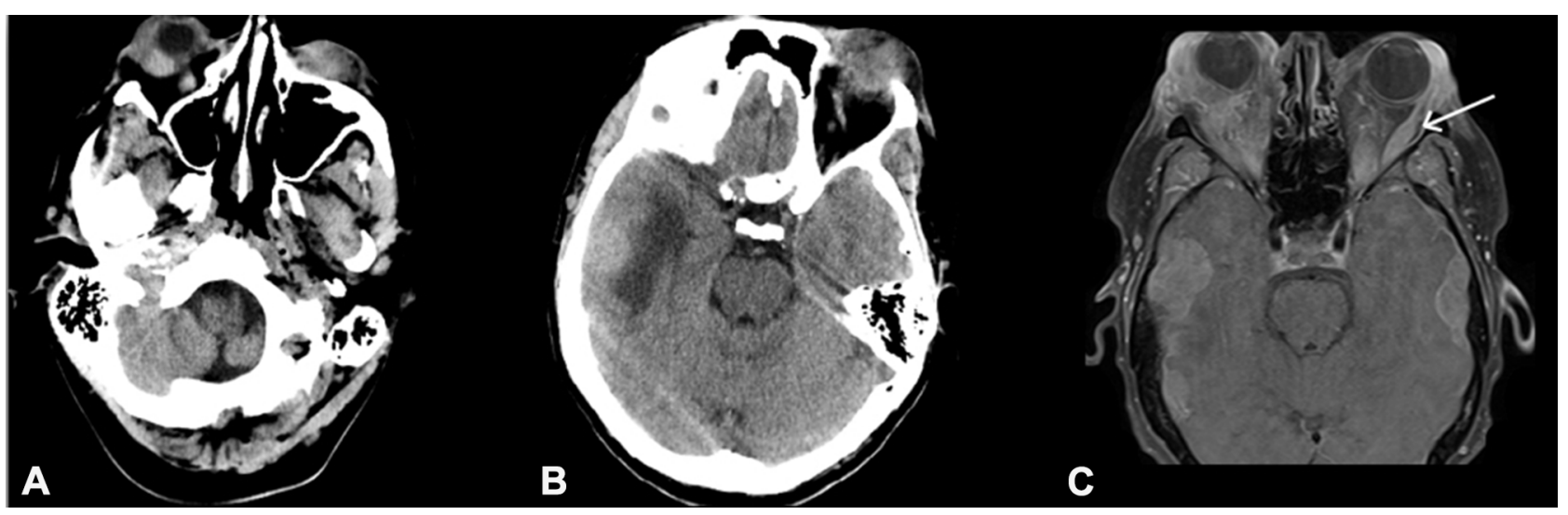

Figure 2 (A, B) Computed tomography showing marked bilateral orbital soft tissue swelling and proptosis, right greater than left, with right retro-bulbar hematoma and/or collection exerting mass effect on the right globe. Bone is intact across both slides cuts. (C) Magnetic resonance imaging demonstrating enhancing intraorbital masses, bilateral proptosis, mass effect, and involvement of extraocular muscles. The left lateral rectus muscle is enlarged (arrow). 
orbital metastases may have lower survival rates as compared to patients with other extramedullary plasmacytomas ${ }^{2}$ Orbital involvement may also indicate insufficient chemotherapy treatment in those who have a diagnosis of multiple myeloma. ${ }^{16}$ This may be a manifesting feature in our present case, as the patient received multiple chemotherapy regimens for relapses since his initial diagnosis. Yet, orbital manifestations are still uncommon, even in adequately treated and relapsed patients, and therefore leads to speculation as to why these orbital tumors may form. Tumor phenotypic variability may be a factor, but further studies should be conducted comparing orbital plasmacytomas and other extramedullary plasmacytomas, and assessing histological and genetic findings for similarities. This may lead to more targeted and definitive treatment therapies.

Another reason for these comparisons is the concept of sanctuary sites in multiple myeloma. Sanctuary sites are present in the brain, central nervous system, male genital tract, and eyes. ${ }^{17}$ These sites provide a "sanctuary" for hematologic malignancy because of blood barriers. Therefore, hematologic malignant cells may remain in the site, even after systemic anticancer chemotherapy for a previous hematologic malignancy such as multiple myeloma. ${ }^{18}$ Testicular plasmacytoma itself is a rare entity, and does not tend to be a primary tumor, but rather a secondary manifestation. ${ }^{19}$ In our patient, it is possible that the testicular relapse that occurred in his disease process due to a sanctuary site phenomenon, and it would apply for the tumor appearing in his orbit as well. Our patient's monoclonal antibodies were IgG lambda, which is different from Yamashita's described IgD lambda-monoclonal antibody, but further analyses should be done to see comparisons between these extramedullary tumors to determine if they are derived from the same sequelae. ${ }^{18}$

It is important for physicians to recognize that a patient presenting with proptosis may be having symptoms of a multiple myeloma relapse in the right clinical context. Further studies should be done determining whether these locations, such as the brain, testicle, and eye, are subject to sanctuary site effects by comparing the pathology of the tumors. When possible, biopsy is the mainstay for definitive diagnosis and highlights the utility of cytology in these lesions.

\section{Ethical Considerations}

Due to the nature of this report, the text has been appropriately deidentified and no information is provided that can be used to identify the subject. Informed written consent has been provided in order to publish the details, images, and text of this script from the next of kin. Institutional approval was not required to publish case details.

\section{Disclosure}

There is no financial support to report for this manuscript. The authors report no conflicts of interest in this work.

\section{References}

1. Rootman J. Diseases of the Orbit: A Multidisciplinary Approach. Philadelphia, PA: Lippincott Williams \& Wilkins; 2003:405-406.

2. Burkat CN, Buren JJV, Lucarelli MJ. Characteristics of orbital multiple myeloma: a case report and literature review. Surv Ophthalmol. 2009;54(6):697-704. doi:10.1016/j.survophthal.2009.04.012

3. Rodman HI, Font RL. Orbital involvement in multiple myeloma. Review of the literature and report of three cases. Arch Ophthalmol. 1972;87(1):30-35. doi:10.1001/archopht.1972.01000020032006

4. Knapp AJ, Gartner S, Henkind P. Multiple myeloma and its ocular manifestations. Surv Ophthalmol. 1987;31(5):343-351. doi:10.1016/ 0039-6257(87)90119-6

5. Malik A, Narang S, Handa U, Sood S. Multiple myeloma presenting as bilateral orbital proptosis. Indian J Ophthalmol. 2009;57 (5):393-395. doi:10.4103/0301-4738.55069

6. Kwartz J, Jackson A, Noble JL. Bilateral proptosis and corneal crystals in multiple myeloma. Eye (Lond). 1993;7(Pt 1):191-193. doi:10.1038/eye. 1993.42

7. Payandeh M, Karami N, Enayati S, Karami A, Aeinfar M, Yari F. Retrobulbar secondary plasmacytoma: a case report and systematic review of the literature. Biomed Res Ther. 2018;5(9):2703-2707. doi:10.15419/bmrat.v5i9.482

8. Thuro BA, Sagiv O, Shinder R, et al. Clinical presentation and anatomical location of orbital plasmacytomas. Ophthalmic Plast Reconstr Surg. 2018;34(3):258-261. doi:10.1097/IOP.00000000 00000939

9. Sharma A, Kaushal M, Chaturvedi NK, Yadav R. Cytodiagnosis of multiple myeloma presenting as orbital involvement: a case report. Cyto Journal. 2006;3:19. doi:10.1186/1742-6413-3-19

10. Ajithkumar TV, Sivasankar C, Ramachandran K. Orbital multiple myeloma: case report and review of computed tomography features. Australas Radiol. 2002;46(1):119-120. doi:10.1046/j.1440-1673. 2001.00974.x

11. Henderson JW, Farrow GM. Orbital Tumors. New York: ThiemeStratton; 1980.

12. Lasocki A, Furphy E, Westerman DA, Harrison S. Retinal ischemia due to extramedullary plasmacytomas of the orbit. J Clin Neurosci. 2020;72:447-449. doi:10.1016/j.jocn.2020.01.052

13. Shinder R, Al-Zubidi N, Esmaeli B. Survey of orbital tumors at a comprehensive cancer center in the United States. Head Neck. 2011;33(5):610-614. doi:10.1002/hed.21498

14. Adkins JW, Shields JA, Shields CL, Eagle RC, Flanagan JC, Campanella PC. Plasmacytoma of the eye and orbit. Int Ophthalmol. 1996;20(6):339-343. doi:10.1007/bf00176888

15. Fung S, Selva D, Leibovitch I, Hsuan J, Crompton J. Ophthalmic manifestations of multiple myeloma. Ophthalmologica. 2005;219:43-48. doi: $10.1159 / 000081782$

16. Kottler UB, Cursiefen C, Holbach LM. Orbital involvement in multiple myeloma: first sign of insufficient chemotherapy. Ophthalmologica. 2003;217(1):76-78. doi:10.1159/000068251 
17. Adalja AA. Sanctuary sites: what lies behind ebola eye infections, sexual transmission, and relapses. Health Security. 2015;13(6):396-398. doi:10. 1089/hs.2015.28999.aaa

18. Yamashita K, Horiuchi T, Hayashida A, et al. Multiple myeloma with testicular involvement: a case report. Urol Case Rep. 2019;26:100971. doi:10.1016/j.eucr.2019.100971
19. Khan M, Rajarubendra N, Azer S, et al. Plasmacytoma of the testis in a patient with relapsed and refractory multiple myeloma: case report and review of the literature. Urol Ann. 2015;7(4):530. doi:10.4103/ 0974-7796.164844

\section{Publish your work in this journal}

The International Medical Case Reports Journal is an international, peer-reviewed open-access journal publishing original case reports from all medical specialties. Previously unpublished medical posters are also accepted relating to any area of clinical or preclinical science. Submissions should not normally exceed 2,000 words or 4 published pages including figures, diagrams and references. The manuscript management system is completely online and includes a very quick and fair peer-review system, which is all easy to use. Visit http://www.dovepress.com/testimonials.php to read real quotes from published authors. 